\title{
Upper extremity musculoskeletal pain among office workers in three Spanish-speaking countries: findings from the CUPID study
}

\author{
Adriana Campos-Fumero, ${ }^{1,2}$ George L Delclos, ${ }^{1,3,4}$ David I Douphrate, ${ }^{1}$ \\ Sarah A Felknor, ${ }^{1,5}$ Sergio Vargas-Prada, ${ }^{3,4}$ Consol Serra, ${ }^{3,4,6}$ David Coggon, 7,8 \\ David Gimeno Ruiz de Porras 1,3,4
}

\begin{abstract}
${ }^{1}$ The University of Texas Health Science Center at Houston, School of Public Health, Houston, Texas, USA ${ }^{2}$ Instituto Tecnológico de Costa Rica, Cartago, Costa Rica ${ }^{3}$ Center for Research in Occupational Health (CiSAL), Universitat Pompeu Fabra, Barcelona, Spain

${ }^{4}$ CIBER Epidemiología y Salud Pública (CIBERESP), Spain

${ }^{5}$ National Institute for Occupational Safety and Health (NIOSH), Atlanta, USA ${ }^{6}$ Department of Occupational Health, Parc de Salut Mar, Barcelona, Spain

${ }^{7}$ Arthritis Research-UK/MRC

Centre for Musculoskeletal Health and Work, University of Southampton, Southampton, UK

${ }^{8}$ Medical Research Council Lifecourse Epidemiology Unit, University of Southamptom, Southampton, UK
\end{abstract}

\section{Correspondence to} Dr Adriana Campos-Fumero, Instituto Tecnológico de Costa Rica, Escuela de Ingeniería en Seguridad Laboral e Higiene Ambiental, Apartado Cartago 159-7050, Costa Rica; acampos@itcr.ac.cr

Received 18 September 2015 Revised 25 January 2016 Accepted 5 February 2016

\begin{tabular}{l} 
To cite: Campos-Fumero A, \\
Delclos GL, Douphrate DI, \\
et al. Occup Environ Med \\
Published Online First: \\
[please include Day Month \\
Year] doi:10.1136/oemed- \\
2015-103327 \\
\hline
\end{tabular}

\section{ABSTRACT}

Objectives To estimate the prevalence and incidence of upper extremity musculoskeletal pain (UEMP) and related disability among office workers in Costa Rica, Nicaragua and Spain.

Methods Data from the multinational Cultural and Psychosocial Influences on Disability (CUPID) study on 947 (93\%) participants at baseline with 90\% follow-up after 12 months were employed. Logistic regression was used to estimate the associations (ORs and corresponding 95\% Cls) between country and six outcomes: baseline prevalence of (1) UEMP in past 12 months, (2) UEMP in past month and (3) disabling UEMP in past month; (4) incidence of new UEMP at follow-up; (5) incidence of new disabling UEMP at follow-up and (6) persistence of UEMP at follow-up, after adjustment for sociodemographic, job-related and health-related covariates.

Results Baseline prevalence of UEMP in the past month was higher in Costa Rica (53.6\%) $(\mathrm{OR}=1.89$; $95 \% \mathrm{Cl} 1.36$ to 2.62 ) and Nicaragua (51.9\%) $(\mathrm{OR}=1.74 ; 95 \% \mathrm{Cl} 1.28$ to 2.35$)$ than in Spain $(38.4 \%)$. Compared to Spain (33.2\%), the incidence of new UEMP was $50.4 \%$ in Costa Rica (OR=2.04; $95 \%$ $\mathrm{Cl} 1.34$ to 3.12 ) and $60.2 \%$ in Nicaragua ( $O R=3.04$; $95 \% \mathrm{Cl} 2.06$ to 4.50). The incidence of disabling UEMP was higher in Nicaragua ( $\mathrm{OR}=2.57 ; 95 \% \mathrm{Cl} 1.50$ to 4.41) and Costa Rica (OR=2.16; $95 \% \mathrm{Cl} 1.22$ to 3.84) when compared to Spain.

Conclusions Prevalence of UEMP was approximately twofold higher and its incidence twofold to threefold higher in Costa Rica and Nicaragua as compared with Spain. Between-country differences were only partially explained by the covariates analysed. Research is needed to explore other aspects of work and cultural attributes that might explain the residual differences in UEMP.

\section{BACKGROUND}

Musculoskeletal disorders (MSDs) are a major cause of morbidity and disability, ${ }^{1}$ and have been identified as the most common work-related health problem, affecting tens of millions of workers globally. ${ }^{2}$ The impact of MSDs can vary from mild symptoms to major functional impairment ${ }^{3}$ with reduced quality of life, ${ }^{4}{ }^{5}$ lower productivity at work, including lost time, ${ }^{6}$ and increased medical expenses and costs due to disability. ${ }^{3}{ }^{4}$ MSDs are

\section{What this paper adds}

- In Latin America, there is a lack of reliable data on working conditions and their effect on workers' health, particularly on highly prevalent problems such as musculoskeletal health among office workers.

- Most research in musculoskeletal pain has been conducted in Western countries, with hardly any research in Central America, so generalisability of findings to low-income and middle-income countries such as Costa Rica and Nicaragua is uncertain.

- This study examines the prevalence and incidence of upper extremity musculoskeletal pain and associated disability among office workers in Nicaragua and Costa Rica, and compares the findings to those from workers performing similar jobs in Spain, a high-income country with a shared language and cultural similarities.

- The results provide valuable information that is lacking in Latin America and that should increase the awareness of the role of working conditions on musculoskeletal health as well as support the development of interventions to reduce musculoskeletal health problems among office workers in Central America.

considered a high-cost national health problem in the USA, ${ }^{7}$ accounting for more than one-third of reported work-related illness, ${ }^{4} 8$ and estimated to affect over 500000 employees each year. ${ }^{9}$ While MSDs occur in workers from all employment sectors, the rapid incorporation of information technology in the workplace has meant that many jobs, in particular office jobs, now involve exposure to perceived risk factors for upper extremity MSDs, such as poor body posture, prolonged sitting and repetitive motions. These may partly explain the high prevalence of upper extremity MSDs in various parts of the world. ${ }^{4}$

Upper extremity musculoskeletal pain (UEMP) is common in office environments worldwide. ${ }^{10} 11$ The availability of statistical data, however, is uneven, and most findings come from high-income 
economies, such as the European Union member states and the USA. For instance, European data have indicated a 1-year prevalence for neck/upper-limb pain of $41.3 \%$ among office clerks. ${ }^{12}$ However, data from low-income and middle-income countries, such as those in the Central America region, are largely lacking. ${ }^{13}{ }^{14}$ Recently, the first Central American Survey of Working Conditions and Health-reported high exposure to repetitive movements ( $>43 \%)$, including in Costa Rica and Nicaragua, but without distinguishing by occupation. ${ }^{14}$

The aetiology of MSDs, and by extension that of UEMP, is multifactorial. $^{4} 1516$ Work-related risk factors associated with the onset of MSDs include physical and psychosocial determinants, ${ }^{5} 6^{17}$ as well as individual characteristics. ${ }^{15} 18$ Psychological and social factors, ${ }^{15}{ }^{19}$ including the role of culture, ${ }^{20}$ are increasingly being considered important contributing factors in MSDs and related disability. ${ }^{21-23}$ However, it is not clear whether these risk factors explain differences in the occurrence of upper extremity MSDs between high-income and low/middle-income economies among specific occupational groups such as office workers. $^{24}$

The aim of this study was to estimate the prevalence and incidence of UEMP and associated disability among office workers in two Spanish-speaking middle-income economies, Costa Rica and Nicaragua, ${ }^{25}$ and compare them to a similar group of office workers also in a Spanish-speaking country, but one with a highincome economy, such as Spain. ${ }^{25}$ In addition, we examined the extent to which sociodemographic characteristics, aspects of employment and working conditions, and health-related variables, explained any differences that were observed among the three countries.

\section{METHODS}

\section{Study design and participants}

We focused on office workers in Costa Rica, Nicaragua and Spain, using data collected as part of Cultural and Psychosocial Influences on Disability (CUPID), an international longitudinal study, ${ }^{20}$ that explores the influence of culturally determined health beliefs and other psychosocial factors on MSDs, and associated disability. In each of the three countries, a minimum of 200 office workers who used computers regularly were randomly sampled from payroll records, and two rounds of interviews were conducted, at baseline and after 12 months (follow-up). In Costa Rica, the sample was recruited from the central offices of the Costa Rican Social Security System, between March 2009 and July 2011. In Nicaragua, participants were recruited between February 2008 and November 2010, from among employees of the Ministry of Labor and Nicaraguan Institute of Social Security. In Spain, data collection occurred between November 2007 and February 2010, among workers from four hospitals and a public university in Barcelona. ${ }^{26}$ Detailed information about the data collection and sample characteristics can be found elsewhere. ${ }^{20}$ Ethical review and approval were obtained prior to data collection, from institutional review committees in each country, and from the University of Texas Health Science Center Committee for the Protection of Human Subjects.

Initial participation rates were $91 \%$ in Costa Rica, $100 \%$ in Nicaragua and $98 \%$ in Spain. ${ }^{20}$ After elimination of 73 participants who did not meet the inclusion criteria (20-59 years of age and worked in the current job for at least 1 year), the final baseline sample comprised 947 (93\%) participants. These included 224 in Costa Rica, 285 in Nicaragua and 438 in Spain. Participation at follow-up $(n=853)$ was $92 \%$ in Costa Rica, $89 \%$ in Nicaragua and $90 \%$ in Spain.
Although there were two other Spanish-speaking countries who were part of the CUPID study (ie, Ecuador and Colombia), we did not include them in our study for reasons other than their official language. Colombia was excluded since only baseline data were collected. Ecuador had follow-up data but was excluded since, despite sharing language similarities, Ecuador is geographically located in South America and differs from Costa Rica and Nicaragua in important aspects. For instance, historically, Ecuador has been more linked to Colombia than to Central America, affecting its cultural and socioeconomic development.

\section{Questionnaire}

The interviewer-administered questionnaires, both at baseline and at follow-up, were first developed in English ${ }^{20}$ and then translated into Spanish with independent back translation to detect misinterpretations. ${ }^{26}$ Additional adjustments to local terminology were made to ensure better understanding of the questions by the respondents. The questionnaires were pilottested in each country in workers similar to the intended study population, but these workers were not included in the final sample.

The baseline questionnaire provided information about sociodemographic characteristics (age, sex, education, height and dominant hand), health-related behaviours (eg, smoking habits) and aspects of work (occupation, duration of employment in current job, physical activities in an average working day, job control, social support, job satisfaction and job security). Sections on pain asked about pain in different anatomical regions, including the shoulder, elbow and wrist/hand in the past month, and past 12 months, using standardised Nordic Questionnaire-style diagrams ${ }^{27}$; whether pain had made specified daily tasks difficult or impossible during the past month; awareness of other people with upper limb pain; beliefs about the causes and consequences of upper limb pain (fear-avoidance beliefs), adapted from the Fear Avoidance Beliefs Questionnaire $^{28}$; awareness of the term, repetitive strain injury (or equivalent); mental health (using questions from the Short Form-36 ${ }^{29}$ ); and distress from common somatic symptoms (using questions from the Brief Symptom Inventory ${ }^{30}$ ). The follow-up questionnaire again asked about pain during the past month, and whether it had been disabling for daily living tasks.

\section{Outcomes}

The outcome of interest was UEMP. Dichotomous variables were created indicating whether pain was present or absent at one or more of six upper limb body sites (right and left shoulder, elbow and wrist/hand); sample size limited our ability to analyse each body site separately. According to anatomical site, pain was classified as disabling if it had interfered with specified daily activities such as combing or brushing hair, bathing/showering, getting dressed, opening bottles, jars, or taps, writing, and locking and unlocking doors. As in previous CUPID studies, ${ }^{31}$ responses to this question were coded as 1 if it was difficult or impossible to perform the activity and 0 if no difficulties were reported.

We created six outcome measures: baseline prevalence of (1) UEMP in the past 12 months; (2) UEMP in the past month; (3) disabling UEMP in the past month; (4) incidence of new UEMP at the 12-month follow-up among participants who initially were free from the symptom; (5) incidence of new disabling UEMP at follow-up in participants who did not have UEMP at baseline and (6) persistence of UEMP at follow-up in those who had UEMP in the past month at baseline. 


\section{Independent variable and covariates}

The main independent variable was country (Costa Rica, Nicaragua and Spain), with Spain as the reference. The covariates examined were: (1) sociodemographic characteristics (sex, age, age when full-time education was completed, participant's dominant hand and height); (2) employment-related characteristics (years in current job, number of hours worked per week, contract type and other jobs); (3) working conditions, including physical job demands over a typical work shift (use of a keyboard $>4 \mathrm{~h}$, other repeated wrist/hand movements $>4 \mathrm{~h}$, repeated elbow bending $>1 \mathrm{~h}$, work with hands above shoulder height $>1 \mathrm{~h}$, lifting $25 \mathrm{~kg}$ (56 lbs) by hand, kneeling or squatting $>1 \mathrm{~h}$, and climbing up or down $>30$ flights of stairs) and a list of psychosocial job conditions including those in the CUPID baseline questionnaire based on items typically used when investigating psychosocial work risk factors: incentives, bonus payments, time pressure, lack of choice, lack of support, job dissatisfaction and perception of job insecurity.

In addition, there was a section on health-related variables, including adverse beliefs regarding causes of UEMP (classified as present if the participant completely agreed that such pain is commonly caused by people's work), beliefs that physical activity is harmful (classified as present if the participant completely agreed that, in people with UEMP, physical activity should be avoided and rest is needed to improve), beliefs that UEMP has a poor prognosis (classified as present if the participant completely agreed that neglecting such pain can cause permanent health problems, and completely disagreed that it usually gets better within 3 months), awareness of 'repetitive strain injury' or similar terms, awareness of someone outside work with UEMP, somatising tendency (number of distressing somatic symptoms in past week, categorised as 0,1 or $\geq 2$, from faintness or dizziness, chest pains, nausea or upset stomach, difficulty breathing, numbness or tingling, feeling weak in parts of the body and hot or cold spells) and mental health (dichotomised as good or intermediate/poor according to a previously defined classification $^{20}$ ). Owing to small cell numbers, the covariates of hours worked per week (most participants worked 31-49 h) and contract type (most participants had a permanent contract) could not be investigated for their effects on the outcomes.

\section{Statistical analysis}

We used unconditional logistic regression to calculate ORs and their corresponding 95\% CIs for associations between independent variables and the UEMP outcomes. For each outcome, we followed Hosmer and Lemeshow's ${ }^{32}$ model-building guidelines. Following Amick et al, ${ }^{33}$ and due to the large number of variables, we selected variables for the final models by groups (sociodemographic, employment, health-related and working conditions). First, we selected variables with a $p$ value $<0.25$ for the association with the outcome of interest in bivariate analyses. Next, we created two multivariable models: (1) an individual-level model, including all the variables selected from step 1 regarding sociodemographic characteristics, employment and health and (2) an organisational-level model, including all of the variables selected from step 1 regarding working conditions. All variables with a $\mathrm{p}$ value $<0.10$ in these analyses were then combined into a single multivariable model. Variables with a $\mathrm{p}$ value $<0.05$ (table 1 ) were retained and adjusted for when assessing the differences in prevalence and incidence between countries. Hosmer and Lemeshow's goodness-of-fit tests of the final models had $\mathrm{p}$ values $>0.80$, except one with $\mathrm{p}=0.50$, but all of them indicating a good fit of the final models. ${ }^{32}$ Analyses were performed with Stata V. 13 (StataCorp, 2013. Stata statistical software: Release 13).

\section{RESULTS}

Table 1 summarises the characteristics of participants by country. More than three-fifths in all three countries were female, with increasing percentages in office workers, ranging from $63 \%$ in Costa Rica and $73 \%$ in Nicaragua to $84 \%$ in Spain. Most participants were $<50$ years old, although the group of individuals aged 20-29 years was about half the size in Spain (17\%) than in Costa Rica and Nicaragua (33\%). In all three countries, a large majority worked 31-49 h/week (ie, full time), although in Spain there was a larger group working $30 \mathrm{~h} /$ week or less $(12 \%)$ than in Nicaragua $(5 \%)$ or Costa Rica (1.4\%). In Costa Rica, about $5 \%$ worked 50 h or more versus only $1.4 \%$ in Nicaragua, and virtually nobody in Spain $(0.2 \%)$. Having a permanent contract was the most common type of contract (over $80 \%$ in all countries), although temporary contracts were not infrequent in Costa Rica (15\%) and Spain (20\%).

Regarding psychosocial working conditions, in Costa Rica and Nicaragua, over three-quarters of the participants reported working under time pressure as compared with only $54 \%$ in Spain; in Nicaragua, 40\% reported lack of support from supervisors and coworkers, whereas fewer reported this exposure in Costa Rica (27\%) and Spain (21\%). There were also country differences in adverse health beliefs regarding UEMP. Twelve per cent of Costa Rican participants believed that upper limb pain is commonly caused by work, but in Spain this percentage was $20 \%$ and in Nicaragua 32\%. Only 3\% of participants in Costa Rica thought that physical activity should be avoided and that rest is needed to recover (physical activity is harmful) from UEMP, but percentages were higher in Spain (10\%) and Nicaragua (13\%). This rank order was reversed with the highest proportion of participants in Costa Rica (22\%) reporting that pain does not improve and that neglecting such pain is dangerous (poor prognosis) compared to $15 \%$ in Spain and $9 \%$ in Nicaragua. A somatising tendency was common, especially in Costa Rica and Nicaragua, where $61 \%$ and $58 \%$ of participants reported distress from two or more somatic symptoms versus only $40 \%$ in Spain. Finally, while in Spain, 24\% participants reported intermediate/poor mental health, percentages were higher in Costa Rica (30\%) and Nicaragua (36\%).

Table 2 shows the crude and adjusted ORs for the prevalence of upper extremity pain and disabling pain at baseline. Crude ORs, as compared to Spain, were between 1.67 (95\% CI 1.18 to 2.36) for prevalence of disabling pain in Costa Rica to 2.14 (95\% CI 1.57 to 2.93) for prevalence of UEMP in the past 12 months in Nicaragua. After adjustment for relevant covariates (see table 2 footnotes), only the 12-month (OR=1.93 (1.31 to 2.84 ) in Costa Rica and $\mathrm{OR}=1.78$ (95\% CI 1.24 to 2.57$)$ in Nicaragua) and 1 month (OR $=1.63$ (95\% CI 1.12 to 2.38$)$ in Costa Rica and OR=1.45 (95\% CI 1.03 to 2.03) in Nicaragua) prevalence of pain remained statistically significant. Within the upper extremity, the 1-month prevalence of wrist/hand pain was higher in Nicaragua (39\%) and Costa Rica (38\%) than in Spain (17\%) (data not shown), while the 12-month prevalence was higher in Nicaragua (54\%) than in Costa Rica (46\%) and Spain (23\%). Covariate adjustment in the models for prevalence varied slightly by model (see table 2) but, overall, the following covariates were included in the final models: sex, age, pain commonly caused by people's work, physical activity is harmful, awareness of someone outside work with pain, prognosis, 
Table 1 Sample baseline characteristics by country

\begin{tabular}{|c|c|c|c|c|c|c|}
\hline & \multicolumn{2}{|c|}{ Costa Rica $(n=224)$} & \multicolumn{2}{|c|}{ Nicaragua $(n=285)$} & \multicolumn{2}{|c|}{ Spain $(n=438)$} \\
\hline & $\mathrm{n}$ & Per cent & $\mathbf{n}$ & Per cent & $\mathbf{n}$ & Per cent \\
\hline \multicolumn{7}{|l|}{ Sociodemographic characteristics } \\
\hline \multicolumn{7}{|l|}{ Sex } \\
\hline Male & 84 & 37.5 & 78 & 27.4 & 72 & 16.4 \\
\hline Female & 140 & 62.5 & 207 & 72.6 & 366 & 83.6 \\
\hline \multicolumn{7}{|l|}{ Age (years) } \\
\hline $20-29$ & 73 & 32.6 & 95 & 33.3 & 73 & 16.7 \\
\hline $30-39$ & 64 & 28.6 & 100 & 35.1 & 165 & 37.7 \\
\hline $40-49$ & 56 & 25.0 & 63 & 22.1 & 152 & 34.7 \\
\hline $50-59$ & 31 & 13.8 & 27 & 9.5 & 48 & 11.0 \\
\hline \multicolumn{7}{|l|}{ Employment-related characteristics } \\
\hline \multicolumn{7}{|l|}{ Hours worked per week } \\
\hline$\leq 30$ & 3 & 1.4 & 15 & 5.3 & 51 & 11.6 \\
\hline $31-49$ & 209 & 93.7 & 266 & 93.3 & 386 & 88.1 \\
\hline$\geq 50$ & 11 & 4.9 & 4 & 1.4 & 1 & 0.2 \\
\hline \multicolumn{7}{|l|}{ Contract type } \\
\hline Permanent & 189 & 85.1 & 279 & 98.9 & 352 & 80.4 \\
\hline Temporary & 33 & 14.9 & 3 & 1.1 & 86 & 19.6 \\
\hline \multicolumn{7}{|l|}{ Psychosocial working conditions } \\
\hline Time pressure & 175 & 78.1 & 228 & 80.0 & 238 & 54.3 \\
\hline Lack of support & 60 & 26.8 & 115 & 40.4 & 94 & 21.5 \\
\hline \multicolumn{7}{|c|}{ Health-related variables } \\
\hline \multicolumn{7}{|c|}{ Adverse health beliefs of upper extremity (UE) pain } \\
\hline Commonly caused by people's work & 27 & 12.1 & 92 & 32.3 & 86 & 19.6 \\
\hline Physical activity is harmful & 7 & 3.1 & 36 & 12.6 & 42 & 9.6 \\
\hline Poor prognosis & 49 & 21.9 & 26 & 9.1 & 67 & 15.3 \\
\hline Know someone outside work with UE pain & 108 & 49.5 & 146 & 51.4 & 198 & 45.2 \\
\hline \multicolumn{7}{|l|}{ Somatising tendency (number of symptoms) } \\
\hline 0 & 46 & 20.7 & 76 & 26.8 & 145 & 33.1 \\
\hline 1 & 40 & 18.0 & 43 & 15.1 & 118 & 26.9 \\
\hline$\geq 2$ & 136 & 61.3 & 165 & 58.1 & 175 & 40.0 \\
\hline \multicolumn{7}{|l|}{ Mental health } \\
\hline Good & 156 & 70.0 & 183 & 64.4 & 331 & 75.6 \\
\hline Intermediate/poor & 67 & 30.0 & 101 & 35.6 & 107 & 24.4 \\
\hline
\end{tabular}

somatising tendency, time pressure, lack of support and mental health.

Among the 853 participants who completed the follow-up, 265 had no pain in the upper extremities at baseline, but had developed pain at follow-up (table 3). The incidence of new pain was $33.2 \%$ in Spain, $50.4 \%$ in Costa Rica and $60.2 \%$ in Nicaragua, differences that were statistically significant with adjusted ORs of 2.17 (95\% CI 1.36 to 3.47) and 3.15 (95\% CI 2.05 to 4.83 ), respectively, when compared with Spain. Among 581 subjects without disabling upper limb pain at baseline, 89 reported disabling pain at follow-up, giving incidence rates of 9.9\% in Spain, 19.3\% in Costa Rica and 22.1\% in Nicaragua. Again, adjusted ORs were significantly elevated for Costa Rica and Nicaragua as compared with Spain (ORs of 2.21 (95\% CI 1.20 to 4.04 ) and 2.39 (95\% CI 1.35 to 4.22 ) respectively). Among 388 participants who reported upper limb pain at baseline, $181(47 \%)$ still had pain at follow-up. As compared with Spain, Nicaragua had a higher rate of persistence $(\mathrm{OR}=2.28$; $95 \%$ CI 1.41 to 3.67 ), but in Costa Rica it was lower $(\mathrm{OR}=0.38 ; 95 \%$ CI 0.22 to 0.65$)$, a pattern that did not change with adjustment for covariates. A slightly different set of covariates were included in the final models for incidence and persistence (see table 3) but, overall, models were adjusted for sex, age, awareness of someone outside work with pain, nondisabling pain at baseline, pain commonly caused by people's work, lack of support and somatising tendency.

\section{DISCUSSION}

To the best of our knowledge, this is the first study to examine the prevalence and incidence of UEMP and associated disability among office workers in Nicaragua and Costa Rica, two lowincome and middle-income countries, and to compare the findings with those from workers performing similar jobs in Spain, a high-income country with a shared language and cultural similarities. We found that both the prevalence and incidence of upper extremity pain and disability varied by country, generally showing higher incidence and prevalence in Costa Rica and Nicaragua than in Spain. The between-country differences were mostly independent of several factors such as basic demographic indicators, health-related factors, and employment and working conditions, including physical and psychosocial aspects of work.

There are only a few studies with which our findings can be compared, in particular studies concerning office workers. Previous reports from the CUPID study have indicated significant variation in the prevalence of musculoskeletal pain among office workers performing similar tasks. None of these, 
Table 2 Prevalence of upper extremity musculoskeletal pain by country at baseline

\begin{tabular}{|c|c|c|c|c|c|c|c|c|c|}
\hline & \multicolumn{3}{|c|}{ Prevalence of pain in the past 12 months } & \multicolumn{3}{|c|}{ Prevalence of pain in past month } & \multicolumn{3}{|c|}{ Prevalence of disabling pain } \\
\hline & \multirow{2}{*}{$\begin{array}{l}\text { Per } \\
\text { cent }\end{array}$} & \multicolumn{2}{|l|}{ OR $(95 \% \mathrm{CI})$} & \multirow{2}{*}{$\begin{array}{l}\text { Per } \\
\text { cent }\end{array}$} & \multicolumn{2}{|l|}{ OR $(95 \% \mathrm{Cl})$} & \multirow{2}{*}{$\begin{array}{l}\text { Per } \\
\text { cent }\end{array}$} & \multicolumn{2}{|l|}{ OR $(95 \% \mathrm{Cl})$} \\
\hline & & Crude & Adjusted* & & Crude & Adjustedt & & Crude & Adjusted $¥$ \\
\hline Spain $(n=438)$ & 50.7 & 1 & 1 & 38.4 & 1 & 1 & 25.3 & 1 & 1 \\
\hline Costa Rica $(n=224)$ & 67.0 & 2.03 (1.45 to 2.84$)$ & 1.93 (1.31 to 2.84$)$ & 53.6 & 1.89 (1.36 to 2.62$)$ & 1.63 (1.12 to 2.38$)$ & 36.2 & 1.67 (1.18 to 2.36$)$ & 1.34 (0.89 to 2.01$)$ \\
\hline Nicaragua $(n=285)$ & 68.8 & 2.14 (1.57 to 2.93$)$ & 1.78 (1.24 to 2.57$)$ & 51.9 & 1.74 (1.28 to 2.35$)$ & 1.45 (1.03 to 2.03$)$ & 39.3 & 1.91 (1.38 to 2.63$)$ & $1.40(0.97$ to 2.01$)$ \\
\hline
\end{tabular}

however, have examined upper extremity pain by collapsing data from several upper extremity anatomical sites to provide combined outcome measures. Certainly, even within the same body region, differences in reported musculoskeletal pain might also be found for specific body sites regarding their distribution by country, which was the main goal of our study. Unfortunately, our study sample size limited our ability to analyse the between-country variability for each body site separately. Some studies with larger samples have examined wrist/ hand pain specifically, a subset of upper extremity pain. For example, the 1-month prevalence of wrist/hand pain in Sri Lanka $(8 \%)^{24}$ and Japan (6\%), ${ }^{34}$ two countries on very different economic levels, was much lower than the prevalence in Costa Rica (38\%) and Nicaragua (39\%), whereas the 1-year prevalence of wrist/hand pain in New Zealand $(33 \%)^{35}$ was similar to that in Costa Rica (46\%) and Nicaragua (54\%). Other studies have shown that up to $50 \%$ of newly hired computer users who engage in prolonged periods of typing frequently experienced MSD symptoms within the first 12 months of work and that $46 \%$ had musculoskeletal pain in the neck/shoulder and hand/ arm areas after the first month of follow-up, ${ }^{36}$ which accords with our results. Regarding the 1-month prevalence of disabling wrist/hand pain, there was a large variation in rates among countries in the CUPID study-more than 14-fold. ${ }^{37}$ At the lower end of the range were Pakistan (2.2\%) and Japan (2.3\%), Spain was intermediate (12.6\%), and Costa Rica $(27.4 \%)$ and Nicaragua $(31.6 \%)$ were at the upper end of the range. ${ }^{37}$ As for the unexpected lower prevalence of persistent pain in Costa Rica, we are unable to provide any possible and adequate explanation. This result is unclear pending confirmation from other research and may well be spurious.
Regarding the role of potentially confounding factors we found that, as indicated by the variables retained in the various final statistical models, the incidence and persistence of UEMP were related to adverse health beliefs (ie, beliefs that such pain is work-related, tendency to somatise and beliefs on physical activity being harmful), and awareness of others with pain at work. Others have reported ${ }^{34} \quad 35 \quad 38-44$ similar effects. Palmer et $a l^{38}$ found that persistence of pain was more common among people who believe pain is caused by their work and expect symptoms to continue being a problem 12 months later.

Tendency to somatise was also associated with UEMP. Our findings regarding the role of somatisation agree with those observed in previous studies. ${ }^{34} 40-43$ In a prospective study by Macfarlane et al, ${ }^{39}$ a somatising tendency was reported to be an important predictor of the onset of forearm pain, and prior studies have also reported that people who tend to worry about common symptoms might be more aware of musculoskeletal pain and be more likely to report it. ${ }^{37}$ In our study, awareness of others with pain at work was also, to some extent, associated with the prevalence and incidence of UEMP. This finding is in agreement with previous reports on disabling wrist and hand pain, such as knowing someone outside work with arm pain and having adverse beliefs (poor prognosis of arm pain), both of which may increase the reported prevalence. ${ }^{37}$

Additionally, our results did not support the idea that higher exposure to physical demands of the job was associated with higher $^{37}$ reports of UEMP. This result should not be a surprise given the homogeneity of exposure in our study sample of office workers (eg, more than $90 \%$ of the participants declared using the keyboard for more than $4 \mathrm{~h} /$ day). This type of homogeneity was, in fact, aimed at by design in the CUPID study to reduce the

Table 3 Incidence and persistence of upper extremity pain by country at follow-up

\begin{tabular}{|c|c|c|c|c|c|c|c|c|c|}
\hline & \multicolumn{3}{|c|}{$\begin{array}{l}\text { Incidence of pain in past month (in } \\
\text { participants* who did not have upper } \\
\text { extremity pain at baseline) }\end{array}$} & \multicolumn{3}{|c|}{$\begin{array}{l}\text { Incidence of disabling pain (in participants* } \\
\text { who did not have upper extremity pain at } \\
\text { baseline) }\end{array}$} & \multicolumn{3}{|c|}{$\begin{array}{l}\text { Persistence of pain (in participants* who had } \\
\text { upper extremity pain the past month at } \\
\text { baseline) }\end{array}$} \\
\hline & \multirow{2}{*}{$\begin{array}{l}\text { Per } \\
\text { cent }\end{array}$} & \multicolumn{2}{|l|}{ OR $(95 \% \mathrm{Cl})$} & \multirow{2}{*}{$\begin{array}{l}\text { Per } \\
\text { cent }\end{array}$} & \multicolumn{2}{|l|}{ OR $(95 \% \mathrm{Cl})$} & \multirow{2}{*}{$\begin{array}{l}\text { Per } \\
\text { cent }\end{array}$} & \multicolumn{2}{|l|}{ OR $(95 \% \mathrm{Cl})$} \\
\hline & & Crude & Adjustedt & & Crude & Adjusted $¥$ & & Crude & Adjusted§ \\
\hline Spain (reference) & \multicolumn{3}{|c|}{ (Cases $=94 / \mathrm{N}=283$ ) } & \multicolumn{3}{|c|}{ (Cases $=29 / \mathrm{N}=292)$} & \multicolumn{3}{|c|}{ (Cases $=69 / \mathrm{N}=152)$} \\
\hline & 33.2 & 1 & 1 & 9.9 & 1 & 1 & 45.4 & 1 & 1 \\
\hline Costa Rica & \multicolumn{3}{|c|}{$($ Cases $=65 / \mathrm{N}=129)$} & \multicolumn{3}{|c|}{ (Cases $=26 / \mathrm{N}=135)$} & \multicolumn{3}{|c|}{ (Cases $=25 / \mathrm{N}=104)$} \\
\hline & 50.4 & $2.04(1.34$ to 3.12$)$ & 2.17 (1.36 to 3.47$)$ & 19.3 & $2.16(1.22$ to 3.84$)$ & $2.21(1.20$ to 4.04$)$ & 24.0 & $0.38(0.22$ to 0.65$)$ & $0.37(0.21$ to 0.68$)$ \\
\hline \multirow[t]{2}{*}{ Nicaragua } & \multicolumn{3}{|c|}{$($ Cases $=106 / \mathrm{N}=176)$} & \multicolumn{3}{|c|}{ (Cases $=34 / \mathrm{N}=154)$} & \multicolumn{3}{|c|}{$($ Cases $=87 / \mathrm{N}=132)$} \\
\hline & 60.2 & $3.04(2.06$ to 4.50$)$ & 3.15 (2.05 to 4.83$)$ & 22.1 & $2.57(1.50$ to 4.41$)$ & 2.39 (1.35 to 4.22$)$ & 65.9 & $2.28(1.41$ to 3.67$)$ & $1.97(1.16$ to 3.35$)$ \\
\hline
\end{tabular}


influence of the physical features in the analysis. Furthermore, we found that psychosocial demands of the job, in particular, time pressure and lack of support, were significant predictors of UEMP. These results also accord with earlier research. ${ }^{40} 4144$

Furthermore, there was evidence of between-country variability with large proportional differences between the highest and lowest percentages. For instance, for the three measures of health beliefs, the difference was of one-third for somatising tendency, about two-thirds for beliefs of pain caused by work and three quarters for beliefs on physical activity being harmful. While these contributed to reducing the magnitude of the differences in UEMP between the three countries under study, they were not enough to totally account for the variability in the UEMP prevalence, incidence and persistence between Costa Rica, Nicaragua and Spain. In the final adjusted models, the between-country differences remained at 1.5 (95\% CI 1.03 to 2.03) for prevalence of pain in the past month in Nicaragua to threefold (95\% CI 2.05 to 4.83 ) for incidence of pain in past month, also in Nicaragua.

The question remains, therefore, what else could explain the differences between the three countries? There could be unmeasured risk factors, such as ergonomic aspects of work, previous occupational exposures (work history) or nonoccupational activities (hobbies, sports, home responsibilities). Beyond self-report of a few work tasks (eg, keyboard use), ergonomic assessments were not included in the study design, so we were unable to examine the role of ergonomic factors assessed by direct observation. ${ }^{44}$ While all study participants performed similar office jobs, ergonomic differences in work settings (eg, not all chairs and work stations are designed identically) might explain some of the between-country differences.

Differences in systems for compensation and financial support of people with work-related MSDs may be another factor in the variability among countries. At the beginning of the study, Costa Rica was thought to have the lowest rate of unemployment $(<5 \%)$, followed by Spain $(5-9 \%)$ and Nicaragua $(10-14 \%){ }^{20}$ At follow-up (2010) (ie, in the midst of the worldwide economic recession), the unemployment rate in Costa Rica (7\%) was higher than that reported at the beginning of the study, while it was lower in Nicaragua (8\%) but substantially higher ${ }^{45}$ in Spain (20\%).

Spain provides more benefits to citizens with health-related incapacity for work than Costa Rica or Nicaragua do. For example, Spain offers social security provisions for unemployment, whereas the other two countries do not. Regarding illhealth retirement, Costa Rica usually provides financial support; Spain does sometimes and Nicaragua does not. All countries provide compensation for work-related MSDs and sick pay in the first 3 months of absence. ${ }^{20}$ There are no major differences in the availability of healthcare for MSDs, insofar as all three countries offer free access to primary care, hospital and other practitioners, either through the employer or external services for insured employees. However, there could be important differences related to the quality of the healthcare services provided for diagnosis and management of MSDs, partly suggested by major variations in total expenditures on health per capita (Nicaragua \$144, Costa Rica \$951 and Spain \$2808). ${ }^{46}$ While differences in healthcare are unlikely to explain variations in the incidence of pain, they may contribute to differences in its persistence and resulting disability. Data on the tests and treatments provided for UEMP in each country are not directly available, and further studies would be needed to explore this.

The strengths of this study were its longitudinal design, the high response rates at baseline (>96\%) and follow-up
(>90.2\%), and the collection of data by standardised questions from validated instruments with predictive validity and acceptable reliability. ${ }^{20}$ Its limitations include the possibility of selection bias due to a healthy worker effect if, for example, patients with UEMP were absent on sick leave at the time the surveys were collected. Information bias could have occurred from differences in the way symptoms were interpreted and understood in different cultures, ${ }^{37}$ but the use of diagrams to illustrate the anatomical sites of interest is likely to have reduced such bias. Another possible source of measurement error was the reliance on participants' recall of symptoms and disability, which were not confirmed by physical examination or clinical assessment, and may not always have been valid. In addition, while the study sample consisted of workers, the outcomes were not specifically work related. In particular, disabling pain reflected difficulty or impossibility in performing certain activities of daily life, but not occupational tasks. Nevertheless, if, for example, a person has difficulty dressing, it is likely that there will also be impacts on their job performance. Finally, although the office worker sample in our study was intended to represent workers regularly performing tasks involving computer use, full representation may have not been accomplished given unmeasured differences in office working conditions (eg, workstation ergonomic features), work history and non-occupational activities.

Other limitations that may have influenced our findings relate to the parent CUPID study. On the one hand, as in any multinational research, there are challenges with translation and cultural adaptation to local languages, even within the same language, as was the case in our study. There is always some degree of uncertainty regarding how people in different cultural contexts understand and respond to a question, even if the question has been 'perfectly' adapted to the context where the research is to be conducted. On the other hand, and most importantly, research has shown that cultural differences should not be overlooked when examining health disparities. ${ }^{47}$ Thus, while the three countries in our study shared the same language and have similarities in culture, and the CUPID study was intended to explore the impact of cultural and psychosocial influences on musculoskeletal symptoms, it may have failed to include metrics on the potential cultural (eg, based on Hofstede's power distance, uncertainty avoidance, individualism and masculinity dimensions) ${ }^{48}$ as well as societal (eg, social networks and participation or societal trust) ${ }^{49}$ between-country differences, which may have contributed to, as well as helped interpret, the differences in prevalence and incidence of UEMP in our study. Still, these details may be difficult to grasp through quantitative survey research only, so alternative qualitative research methods would be useful to help interpret the complex reality and implications of the data obtained via quantitative research methods.

In summary, information about musculoskeletal pain and its implications for workers' health has received little attention in Latin America, ${ }^{50}$ most research having been conducted in Western countries. To the best of our knowledge, no other studies in the Central American region have estimated the prevalence and incidence of UEMP. This study provides valuable information that can assist in the development of programmes to reduce UEMP among office workers in the region, and in the planning of research to evaluate the impact of such interventions.

Acknowledgements The authors thank the University of Southampton coordinating team, all coordinators in Nicaragua, Costa Rica and Spain, all other coordinators, data collectors, all the organisations that allowed their employees to participate; and, especially, all the workers who participated in the study. 
Collaborators George L Delclos; David I Douphrate; Sarah A Felknor; Sergio Vargas-Prada; Consol Serra; David Coggon; David Gimeno Ruiz de Porras.

Contributors $A C-F$ is the guarantor of the present article. AC-F, DGRdP and GLD designed the study and DC conceptualised the idea of the parent CUPID study. AC-F and DGRdP planned the statistical analysis and analysed the data. DGRdP, SAF and DC were responsible for supervision of fieldwork, data cleaning and management. DID, SV-P and CS helped in the interpretation of the results. All the co-authors contributed sufficiently to the manuscript and approved the final version.

Funding Funding for this research was supported by grant number $5 \mathrm{~T} 42 \mathrm{OH} 008421$ from the National Institute for Occupational Safety and Health (NIOSH)/Centers for Disease Control and Prevention (CDC) to the Southwest Center for Occupational and Environmental Health (SWCOEH), a NIOSH Education and Research Center, Fogarty grant number 5D43TW000644-13 and Colt Foundation CF/03/05.

Competing interests None declared.

Patient consent Obtained.

Ethics approval The University of Texas Health Science Center Committee for the Protection of Human Subjects.

Provenance and peer review Not commissioned; externally peer reviewed.

\section{REFERENCES}

1 Parent-Thirion A, Macias E, Hurley J, et al. Fourth European Working Conditions Survey. Dublin: European Foundation for the Improvement of Living and Working Conditions, 2007.

2 Niu S. Ergonomics and occupational safety and health: an ILO perspective. App/ Ergon 2010;41:744-53.

3 Rempel DM, Harrison RJ, Barnhart S. Work-related cumulative trauma disorders of the upper extremity. JAMA 1992;267:838-42.

4 Punnett L, Wegman DH. Work-related musculoskeletal disorders: the epidemiologic evidence and the debate. J Electromyogr Kinesiol 2004;14:13-23.

5 Meijer EM, Sluiter JK, Frings-Dresen MH. Is workstyle a mediating factor for pain in the upper extremity over time? J Occup Rehabil 2008;18:262-6.

6 Haufler AJ, Feuerstein M, Huang GD. Job stress, upper extremity pain and functional limitations in symptomatic computer users. Am J Ind Med 2000;38:507-15.

7 National Research Council (US). Panel on Musculoskeletal Disorders, the Workplace and Institute of Medicine (US). Musculoskeletal Disorders and the Workplace: Low Back and Upper Extremities. Washington DC: National Academies Press, 2001.

8 Bernard B ed. Musculoskeletal disorders and workplace factors: a critical review of epidemiologic evidence for work-related musculoskeletal disorders of the neck, upper extremity, and low back. DHHHS (NIOSH) publication no.97-141. Cincinnati, OH: U.S. Department of Health and Human Services, Centers for Disease Control and Prevention. National Institute for Occupational Safety and Health, 1997.

9 Tanaka S, Petersen M, Cameron L. Prevalence and risk factors of tendinitis and related disorders of the distal upper extremity among US workers: comparison to carpal tunnel syndrome. Am J Ind Med 2001;39:328-35.

10 Gerr F, Letz R, Landrigan PJ. Upper-extremity musculoskeletal disorders of occupational origin. Annu Rev Public Health 1991;12:543-66.

11 Gerr F, Marcus M, Monteilh C. Epidemiology of musculoskeletal disorders among computer users: lesson learned from the role of posture and keyboard use. $J$ Electromyogr Kinesiol 2004;14:25-31.

12 Farioli A, Mattioli S, Quaglieri A, et al. Musculoskeletal pain in Europe: the role of personal, occupational, and social risk factors. Scand I Work Environ Health 2014:40:36-46.

13 Coury HJ. Time trends in ergonomic intervention research for improved musculoskeletal health and comfort in Latin America. Appl Ergon 2005:36:249-52.

14 Benavides FG, Wesseling C, Delclos GL, et al. Working conditions and health in Central America: a survey of 12,024 workers in six countries. Occup Environ Med 2014;71:459-65.

15 Bongers PM, de Winter CR, Kompier MAJ, et al. Psychosocial factors at work and musculoskeletal disease. Scand I Work Environ Health 1993;19:297-312.

16 Wahlstrom J. Ergonomics, musculoskeletal disorders and computer work. Occup Med 2005:55:168-76.

17 Feuerstein M, Nicholas RA, Huang GD, et al. Job stress management and ergonomic intervention for work-related upper extremity symptoms. Appl Ergon 2004;35:565-74

18 Waersted M, Hanvold TN, Veiersted KB. Computer work and musculoskeletal disorders of the neck and upper extremity: a systematic review. BMC Musculoskelet Disord 2010;11:1-15.

19 Marras WS, Cutlip RG, Burt SE, et al. National occupational research agenda (NORA) future directions in occupational musculoskeletal disorder health research. Appl Ergon 2009;40:15-22.

20 Coggon D, Ntani G, Palmer KT, et al. The CUPID (cultural and psychosocial influences on disability) study: methods of data collection and characteristics of study sample. PLOS ONE 2012;7:e39820.
21 Madan I, Reading I, Palmer KT, et al. Cultural differences in musculoskeletal symptoms and disability. Int J Epidemiol 2008;37:1181-9.

22 Coggon D. Occupational medicine at a turning point. Occup Environ Med 2005:62:281-3.

23 Armstrong TJ, Buckle P, Fine LJ, et al. A conceptual model for work-related neck and upper-limb musculoskeletal disorders. Scand I Work Environ Health 1993;19:73-84.

24 Warnakulasuriya S, Peiris-John $\mathrm{R}$, Coggon $\mathrm{D}$, et al. Musculoskeletal pain in four occupational populations in Sri Lanka. Occup Med 2012;62:269-72.

25 The World Bank Group. New country classifications. 07/02/2013;2014: http://data. worldbank.org/news/new-country-classifications-2015.

26 Vargas-Prada S, Serra C, Martínez JM, et al. Psychological and culturally-influenced risk factors for the incidence and persistence of low back pain and associated disability in Spanish workers: findings from the CUPID study. Occup Environ Med 2013;70:57-62.

27 Kuorinka I, Jonsson B, Kilbom A, et al. Standardised Nordic questionnaires for the analysis of musculoskeletal symptoms. Appl Ergon 1987;18:233-7.

28 Waddell G, Newton M, Henderson I, et al. A fear-avoidance beliefs questionnaire (FABQ) and the role of fear-avoidance beliefs in chronic low back pain and disability. Pain 1993;52:157-68.

29 Ware JE Jr, Sherbourne CD. The MOS 36-item short-form health survey (SF-36): I. conceptual framework and item selection. Med Care 1992;30:473-83.

30 Derogatis $L R$, Melisaratos N. The brief symptom inventory: an introductory report. Psychol Med 1983;13:595-605.

31 Vargas-Prada S, Martinez JM, Coggon D, et al. Health beliefs, low mood, and somatizing tendency: contribution to incidence and persistence of musculoskeletal pain with and without reported disability. Scand I Work Environ Health 2013;39:589-98

32 Hosmer DW, Lemeshow S. Applied logistic regression. New York, NY: John Wiley \& Sons, 2000.

33 Amick BC III, Habeck RV, Ossmann J, et al. Predictors of successful work role functioning after carpal tunnel release surgery. I Occup Environ Med 2004:46:490-500.

34 Matsudaira K, Palmer KT, Reading I, et al. Prevalence and correlates of regional pain and associated disability in Japanese workers. Occup Environ Med 2011;68:191-6

35 Harcombe H, McBride D, Derrett S, et al. Prevalence and impact of musculoskeletal disorders in New Zealand nurses, postal workers and office workers. Aust N Z J Public Health 2009:33:437-41.

36 Gerr F, Marcus M, Ensor C, et al. A prospective study of computer users: I. study design and incidence of musculoskeletal symptoms and disorders. Am J Ind Med 2002:41:221-35

37 Coggon D, Ntani G, Palmer KT, et al. Disabling musculoskeletal pain in working populations: is it the job, the person, or the culture? Pain 2013;154:856-63.

38 Palmer KT, Reading I, Linaker C, et al. Population-based cohort study of incident and persistent arm pain: role of mental health, self-rated health and health beliefs. Pain 2008;136:30-7.

39 Macfarlane GJ, Hunt IM, Silman AJ. Role of mechanical and psychosocial factors in the onset of forearm pain: prospective population based study. BMJ 2000;321:676-9

40 Fujii T, Matsudaira K, Yoshimura N, et al. Associations between neck and shoulder discomfort (katakori) and job demand, job control, and worksite support. Mod Rheumatol 2013;23:1198-204.

41 Sadeghian F, Raei M, Ntani G, et al. Predictors of incident and persistent neck/ shoulder pain in Iranian workers: a cohort study. PLOS ONE 2013;8:e57544.

42 Solidaki E, Chatzi L, Bitsios P, et al. Work related and psychological determinants of multi-site musculoskeletal pain. Scand J Work Environ Health 2010;36:54.

43 Solidaki E, Chatzi L, Bitsios P, et al. Risk factors for new onset and persistence of multi-site musculoskeletal pain in a longitudinal study of workers in crete. Occup Environ Med 2013;70:29-34.

44 Coggon D, Ntani G, Palmer KT, et al. Patterns of multisite pain and associations with risk factors. Pain 2013;154:1769-77.

45 The World Bank Group. Unemployment, total (\% of total labor force) (modeled ILO estimate). 07/02/2013;2014: http://data.worldbank.org/indicator/SL.UEM.TOTL.ZS.

46 World Health Organization. Global health expenditure database. 2014;2014: http:// apps.who.int/nha/database/QuickReports/Index/en.

47 Mansyur CL, Amick BC, Harrist RB, et al. The cultural production of health inequalities: a cross-sectional, multi-level examination of 52 countries. Int J Health Serv 2009;39:301-19.

48 Hofstede G, Hofstede G, Minkov M. Cultures and organizations: Software of the Mind. 3rd edn. New York: McGraw-Hill, 2010.

49 Mansyur $\mathrm{CL}$, Amick BC, Franzini L, et al. Culture and the social context of health inequalities. Int J Health Serv 2009;39:85-106.

50 Ortiz-Hernandez L, Tamez-Gonzalez S, Martinez-Alcantara S, et al. Computer use increases the risk of musculoskeletal disorders among newspaper office workers. Arch Med Res 2003;34:331-42. 
Upper extremity musculoskeletal pain among office workers in three Spanish-speaking countries: findings from the CUPID study

Adriana Campos-Fumero, George L Delclos, David I Douphrate, Sarah A Felknor, Sergio Vargas-Prada, Consol Serra, David Coggon and David Gimeno Ruiz de Porras

Occup Environ Med published online February 23, 2016

Updated information and services can be found at:

http://oem.bmj.com/content/early/2016/02/23/oemed-2015-103327

\section{Notes}

\section{These include:}

References This article cites 42 articles, 6 of which you can access for free at: http://oem.bmj.com/content/early/2016/02/23/oemed-2015-103327\#ref -list-1

\section{Email alerting} service

Receive free email alerts when new articles cite this article. Sign up in the box at the top right corner of the online article.

To request permissions go to:

http://group.bmj.com/group/rights-licensing/permissions

To order reprints go to:

http://journals.bmj.com/cgi/reprintform

To subscribe to BMJ go to:

http://group.bmj.com/subscribe/ 